\title{
Setting Equilibrium Prices, Approximately
}

\author{
BRENDAN LUCIER \\ Microsoft Research New England
}

\begin{abstract}
We outline recent results, positive and negative, on pricing indivisible goods to approximately maximize social welfare. We describe a relaxation of standard pricing problems in which a seller can bundle goods together prior to sale.

Categories and Subject Descriptors: J.4 [Computer Applications]: Social and Behavioral Sciences-Economics

General Terms: Algorithms, Economics, Theory

Additional Key Words and Phrases: Combinatorial auctions; Walrasian equilibrium; Envy-free
\end{abstract}

Consider the plight of a retail clothing store owner who must decide how best to sell his wares. The owner takes pride in his store, and wants above all else to distribute his inventory to maximize the welfare of his customers. In principle, the owner could consult with each customer about his or her fashion needs, then ultimately propose a wardrobe and price to each one. While such a business model might be feasible for a super-elite fashion outlet, a more practical (and common) approach is to post prices on individual items and let the customers choose what to purchase at the specified prices. The seller's problem then becomes one of selecting appropriate prices.

In this letter we discuss this classic equilibrium pricing problem, using the clothing store as a running example. We will review some well-known impossibility results, then turn to approximation methods as a way to circumvent them.

First, we must establish what it means to set "appropriate" prices. The common wisdom from freshman-level economics is that prices should equate supply and demand. However, this task is not straightforward in the clothing store setting, where there are many heterogeneous goods and buyers can have idiosyncratic preferences. More concretely, suppose that each item of clothing is an indivisible object, and that each customer has a certain value for any given set of clothes. We will imagine that the store owner is so experienced that he knows the values of all of his customers, and that the customers are rational enough to make the best clothing selection for themselves when faced with a set of prices. Even under these favorable conditions, it is still not immediately obvious how prices should be chosen.

A formal study of such market equilibria goes as far back as Walras in 1874; [Shapley and Shubik 1971] provides a modern market-pricing interpretation. The Walrasian solution concept requires that prices satisfy the following conditions:

(1) Each buyer can obtain his most-demanded set at the given prices, and

(2) Every item with a positive price is sold.

These two conditions define a Walrasian equilibrium (WE). The first is a notion of

Author's address: brlucier@microsoft.com 
envy-freeness: no customer will envy another for getting the very last pair of pants in the store. The second condition concerns the seller, who should not be left with an unsold suit jacket that a customer would have purchased at a lower price.

A wonderful feature of a Walrasian equilibrium is that it necessarily results in a socially optimal allocation [Bikhchandani and Mamer 1997], as desired by the store owner. Unfortunately for the owner, WE do not always exist. As a simple example, suppose the store has a pair of shoes for sale and that each shoe is an item. Further imagine that two customers, Alice and Bob, are interested in shoes. Alice, a typical customer, values the pair of shoes at $\$ 10$ but has no value for either shoe on its own. Bob, on the other hand, wants only a single shoe and will pay up to $\$ 6$ for either the left or the right. In this situation, no vector of prices can satisfy the WE conditions: if Alice buys both shoes, then at least one shoe must have price less than $\$ 6$ and is therefore also desired by Bob, violating the first condition of WE. If instead Bob gets a shoe for $\$ 6$ or less, then the other shoe must be left unsold. The second WE condition implies this unsold shoe must have price $\$ 0$, but then Alice would want to buy the pair.

It is perhaps unsurprising that item prices fail to resolve markets with complementary items (like left and right shoes), but the issue extends even further. [Kelso and Crawford 1982] show that a WE always exists when the buyer preferences satisfy a certain gross substitutes condition, which is strictly stronger than submodularity. However, [Gul and Stacchetti 1999] show that this is as far as the existence guarantee can be extended, in the following natural sense. If there is even one customer with values that do not satisfy the gross substitutes condition, a WE can fail to exist. Thus, even for items that have decreasing marginal value, there may not exist item prices that implement a market equilibrium.

\section{Approximations and Unsold Items}

Faced with these classic and discouraging impossibility results, we propose relaxing the market-clearing requirements of WE. Specifically, we will drop the second WE condition, allowing items to remain unsold, and require only that customers can buy their most desired sets. This certainly addresses the existence problem, since the seller could set prices so high that no customer desires anything in the store. Of course, this is a very unsatisfactory solution from the perspective of maximizing social welfare. We therefore turn to the paradigm of approximation and ask whether there exist prices that result in approximately optimal social welfare.

It is not too hard to see that the answer is no for settings with complements: consider extending the shoe example so that Alice gets value only if she buys many, many items together. Nevertheless, our expectation was that, following recent results in the theory of simultaneous item auctions [Christodoulou et al. 2008; Feldman et al. 2013a], constant factor approximations would be forthcoming when customer preferences are complement-free (or, at least, fractionally subadditive; see [Feige and Vondrak 2006]). However, much to our surprise, this is not the case. There exist simple examples without complements for which item prices fail to achieve non-trivial approximations to the economically efficient outcome. We now describe such an example, from [Feldman et al. 2013b].

In this example, two customers Alex and Betty wish to purchase shirts. Alex 
is not particular about shirts, but could use many of them: he would value any number of shirts at $\$ 1$ each, regardless of style. However, Alex also has a strong preference for not leaving the store empty-handed, and therefore has a value of $\$ 2$ for getting any one shirt. In other words, Alex values any set of $k>1$ shirts at $\$ k$, and values any single shirt at $\$ 2$. Betty is also completely indifferent about style, but is only interested in buying at most one shirt: her value is slightly less than $\$ 1$ for any single shirt, where the precise value will be described below. It is easily checked that both Alex's and Betty's value functions are subadditive (in fact, they satisfy the stronger condition of fractional subadditivity).

To understand the store owner's pricing problem, consider what would happen if Alex were the only customer. The store owner certainly wants Alex to buy as many shirts as possible. It is tempting to set a price of $\$ 1$ on every shirt. At these prices, Alex could indeed buy many shirts for an overall utility (value minus price) of $\$ 0$, but he prefers to buy a single shirt for a gain of $\$ 1$. Thus, in order to convince Alex to buy all of the shirts in the store, the retailer must set a price less than $\$ 1$ on some of them. For example, if the store has $m$ shirts, a price of $\$\left(1-\frac{2}{m}\right)$ on each shirt would convince Alex to buy them all.

This solution falls apart, however, when we include Betty as a customer. If Betty's value for a shirt is sufficiently close to $\$ 1$ - say, $\$\left(1-\frac{1}{m}\right)$ - she competes with Alex for the cheapest shirt. Indeed, Alex only ever buys multiple shirts if their average price is less than Betty's value, so he and Betty must have conflicting demands. The only way to resolve this tension is to allocate at most one shirt to Alex - say, by setting a price of $\$ 2$ on every shirt — which catastrophically reduces the social welfare from $\$ m$ to at most a constant.

\section{The Power of Bundling}

The example above illustrates that allowing items to go unsold is not enough to enable reasonable approximations. We must therefore consider a further relaxation. To motivate our approach, let us return to the example of selling shoes to Alice and Bob. It is common for stores to offer shoes for sale, and the sort of problem present in our example is certainly not insurmountable in practice. Allowing shoes to remain unsold is not the natural approach — quite the opposite! The more obvious solution is to commit to selling shoes only in pairs. This effectively defines a pair of shoes to be an indivisible object. This is a natural power to afford the seller; after all, as the owner of the goods, he is free to package them however he wishes. Furthermore, this type of bundling does not increase the complexity of the marketplace, since the seller is still posting prices on individual, indivisible items. In fact, the market is arguably simpler, since the owner can only reduce the number of items for sale by pairing shoes.

More generally, we could allow the owner of our clothing store to partition his goods into packages however he sees fit, then treat those packages as the items for sale and set their prices. The goal, then, is to find a partition and a set of prices so that the buyer-side envy-freeness condition is met. This solution concept was proposed in [Feldman et al. 2013b], where it was termed Combinatorial Walrasian equilibrium. As it so happens, this additional bundling operation is just what we are looking for: in [Feldman et al. 2013b] we show that, for arbitrary customer 
preferences, there always exists a partition of the objects and a pricing such that the resulting outcome achieves at least half of the optimal social welfare.

To illustrate this approach, let us return to the example of Alex and Betty buying shirts. In this example, the correct choice for the store owner is to commit to selling all of his stock as a single bundle, for a price of $\$ m$. Betty is unwilling to buy at such a high price, and Alex is not tempted to buy only a single shirt (since individual shirts are not for sale). The optimal allocation, where Alex buys everything in the store, becomes the only possible outcome. Effectively, the presence of the insatiable Alex compels the store owner to switch to a wholesale business model.

\section{REFERENCES}

Bikhchandani, S. and Mamer, J. W. 1997. Competitive equilibrium in an exchange economy with indivisibilities. J. of Economic Theory 74, 2, 385-413.

Christodoulou, G., Kovács, A., and Schapira, M. 2008. Bayesian combinatorial auctions. In ICALP. 820-832.

Feige, U. AND VondraK, J. 2006. Approximation algorithms for allocation problems: Improving the factor of 1 - 1/e. In FOCS. 667-676.

Feldman, M., Fu, H., Gravin, N., And Lucier, B. 2013a. Simultaneous auctions are (almost) efficient. In $S T O C$. To appear.

Feldman, M., Gravin, N., ANd Lucier, B. 2013b. Combinatorial walrasian equilibrium. In STOC. To appear.

Gul, F. And Stacchetti, E. 1999. Walrasian equilibrium with gross substitutes. J. of Economic Theory 87, 1, 95-124.

Kelso, Alexander S, J. and Crawford, V. P. 1982. Job matching, coalition formation, and gross substitutes. Econometrica 50, 6, 1483-1504.

Shapley, L. S. And Shubik, M. 1971. The assignment game i: The core. International J. of Game Theory 1, 111-130. 